\title{
A novel mutation in $T T N$ gene in a Saudi patient with bilateral facial weakness and scapular winging
}

\author{
Hussein Algahtani ${ }^{1, *}$, Bader Shirah ${ }^{2}$, Raghad Algahtani ${ }^{3}$, Mohammad H. Al-Qahtani ${ }^{4}$, \\ Angham Abdulrahman Abdulkareem ${ }^{4}$, Muhammad Imran Naseer, \\ ${ }^{1}$ King Abdulaziz Medical City / King Saud bin Abdulaziz University for Health Sciences, Jeddah, Saudi Arabia; \\ ${ }^{2}$ King Abdullah International Medical Research Center/King Saud bin Abdulaziz University for Health Sciences, Jeddah, Saudi \\ Arabia; \\ ${ }^{3}$ King Saud bin Abdulaziz University for Health Sciences, Jeddah, Saudi Arabia; \\ ${ }^{4}$ Center of Excellence in Genomic Medicine Research, King Abdulaziz University, Jeddah, Saudi Arabia; \\ ${ }^{5}$ Department of Medical Laboratory Technology, Faculty of Applied Medical Sciences, King Abdulaziz University, Jeddah, Saudi \\ Arabia.
}

\begin{abstract}
Summary Titin (TTN) is a large gene with 363 exons that encodes a large abundant protein (longest known polypeptide in nature) that is expressed in cardiac and skeletal muscles. TTN has an important role in the sarcomere organization, assembly of muscles, transmission of the force at the Z-line, passive myocyte stiffness, and resting tension maintenance in the I-band region. Mutation in extreme $\mathrm{C}$ terminus of TTN, situated at the end of M-band of the TTN in chromosome 2q31, results in tibial muscular dystrophy (TMD), also called Udd Distal Myopathy, which is an autosomal dominant distal myopathy. In this article, we report a novel mutation in $T T N$ gene in a Saudi patient with bilateral facial weakness and scapular winging. This report adds to the literature a heterozygous missense variant $\mathrm{c} .85652 \mathrm{C}>\mathrm{G}$, p.(Pro28551Arg) in TTN gene, which may be related to genes that cause the disease, but more case validation is needed. The novel mutation described in the present study widened the genetic spectrum of $T T N$-associated diseases, which may benefit studies addressing this disease in the future.
\end{abstract}

Keywords: Titin, TTN, tibial muscular dystrophy, neuromuscular disorders, Saudi Arabia

\section{Introduction}

Titin $(T T N)$ is a large gene with 363 exons that encodes a large abundant protein (longest known polypeptide in nature) that is expressed in cardiac and skeletal muscles. TTN protein has an important role in sarcomere organization, assembly of muscles, transmission of the force at the Z-line, passive myocyte stiffness, and resting tension maintenance in the I-band region (1). Mutation in extreme $\mathrm{C}$ terminus of $T T N$, situated at the end of M-band in chromosome 2q31, results in tibial muscular

Released online in J-STAGE as advance publication May 29, 2019.

*Address correspondence to:

Dr. Hussein Algahtani, King Abdulaziz Medical City/King Saud bin Abdulaziz University for Health Sciences, P.O. Box: 12723, Jeddah 21483, Saudi Arabia. Contact No.: 00966556633130.

E-mail: halgahtani@hotmail.com dystrophy (TMD, MIM\#600334), also called Udd Distal Myopathy. In addition, truncation mutations of TTN are considered the most common cause of familial dilated cardiomyopathy (2). TMD is a mild autosomal dominant distal myopathy involving the anterior compartment muscles of the lower legs (3). TMD was first described in Finnish patients; its prevalence in Finland is estimated to be $>1 / 10,000$. TMD usually begins in adulthood, between ages 35-55 years, and is a slowly progressive, benign distal myopathy typically restricted to the anterior muscles of the lower leg (tibialis anterior, extensor halluces longus, extensor digitorum longus). In some patients, muscle weakness also progresses to proximal lower leg musculature (4).

In this article, we report a novel mutation in TTN gene in a Saudi patient with bilateral facial weakness and scapular winging. What makes this report interesting is not only the novel genetic mutation but also the atypical clinical presentation with lack of distal muscle 
involvement, which is the characteristic mark of this genetic disorder.

\section{Case Report}

A proband III-1, a 34-year-old male presented to the neurology clinic with weakness of the upper extremities and difficulty in performing activities of daily living such as combing his hair and using utensils. He denied swallowing difficulty, visual symptoms, sensory symptoms, cramps, fasciculations, headache, skin lesions, joint symptoms, or oro-genital ulcers. Systemic review and past history were unremarkable. He denied any history of diabetes mellitus, heart disease, malignancy, or connective tissue disorders. Family history was remarkable for a similar disease in all of his three brothers. Parents of the patient are not consanguineous.

His vital signs and general medical examination were normal. Neurological examination showed normal higher mental functions, speech, and cranial nerves II to XII. Motor examination showed winging of the left scapular to medial side and mild facial diplegia. Lower limb examination including tone, power, and reflexes were normal. Coordination and cerebellar functions were normal. Serum creatine kinase (CK) level was elevated at $423 \mathrm{IU} / \mathrm{L}$ (normal 27-132 IU/L), CK-MB isoenzyme at $12.9 \mathrm{IU} / \mathrm{L}$ (normal 5-25 IU/L), and CKMB percentage of $3.05 \%$ (normal 3-5\%). The rest of the blood tests were unremarkable.

Nerve conduction studies were normal. Electrocardiogram and echocardiography were normal. Needle electromyography was performed on the serratus anterior, latissimus dorsi, deltoid, rhomboids major, and rhomboids minor. Spontaneous denervation potentials were not observed. The muscle membrane was stable with normal insertional activity. The motor units in the rhomboids major and minor were relatively large and long with reduced recruitment. The serratus anterior was difficult to sample, likely due to atrophy. The MUAPs in the latissimus dorsi appeared relatively small with relatively shorter duration than the neighboring muscles. The electromyography of the deltoid was unremarkable. These findings reflect a long-standing chronic myopathic process involving the shoulder girdle muscles on the left side, especially the rhomboids and latissimus dorsi with a dropout of motor units due to the chronicity of the myopathic process.

The detailed family pedigree was drawn after getting the detailed information from the parents as shown in figure 1. Given the strong family history, genetic testing was performed using whole exome sequencing. TTN gene mutation identified through exome sequencing, which was further validated by using Sanger sequencing in all members III-1, III-2. IV-1, IV-2, IV-3, IV-4, IV-5 of the family. Primers were designed for the target region, and TTN gene was amplified by polymerase chain reaction (PCR). Purified PCR products were Sanger sequenced by the Big Dye terminator method by using Applied Biosystems 3700 (ABI 3700).

We identified a heterozygous missense variant c.85652C $>$ G, p.(Pro28551Arg) in TTN gene. The variant affects six RefSeq annotated transcripts and is located in exon 326 in the metatranscript (NM_001267550.1) containing a total of 363 exons. The variant has not been observed in large reference population cohorts. The variant is predicted damaging by in silico pathogenicity prediction tools. Furthermore, in the Greater Middle East (GME) variome minor allele frequency was 0.00 in the database. Moreover, PolyPhen 0.7, and SIFT 0.12 , PhyloP and (phyloP46way_placental) predicted as a causative mutation. This mutation was absent in the Human Gene Mutation Database (HGMD, www. hgmd.cf.ac.uk/) MIM. 1000 genome (http://www. internationalgenome.org/) and ExAc (Version 0.3.1)

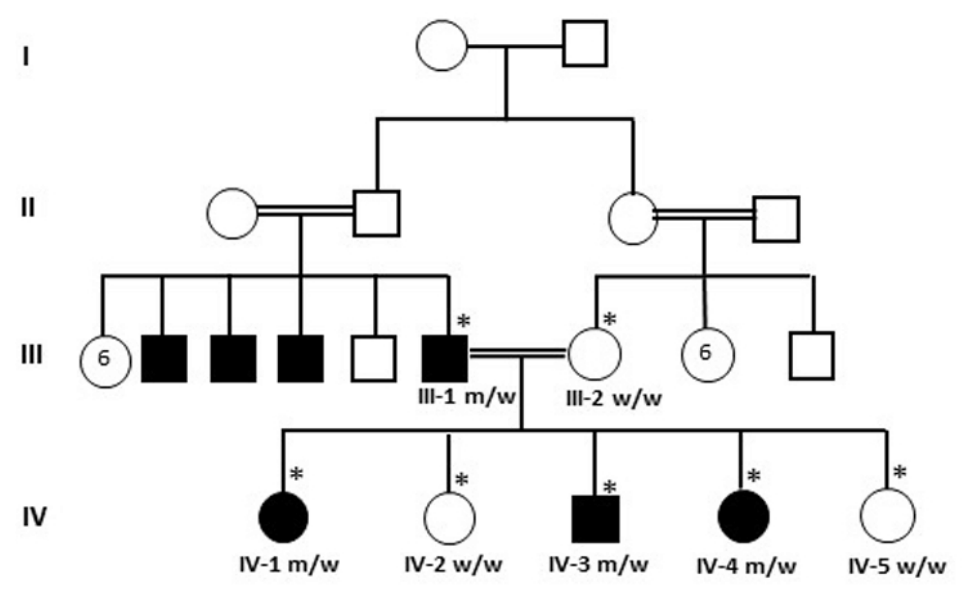

Figure 1. A large consanguineous family from Saudi Arabia showing the disease phenotype. The samples marked with asterisks were available for genetic testing. Blocked square and circles indicate the infected individuals. Mutant (m) and wild-type (wt) allele are marked in the analyzed individuals. 


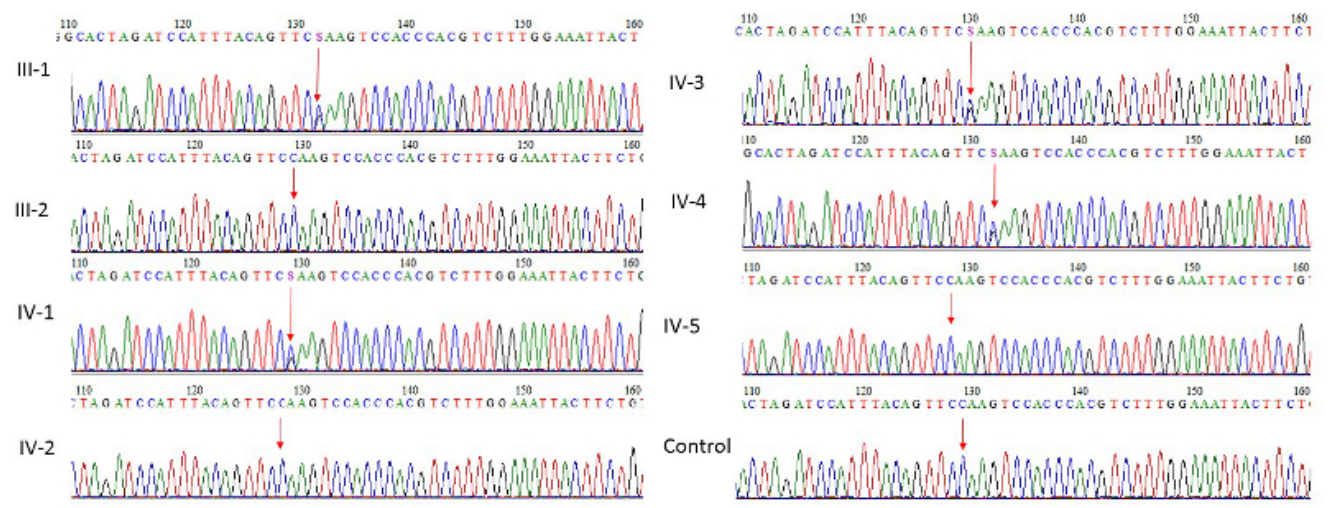

Figure 2. Sanger sequence analysis for validation of exome sequencing. III-2, IV-2, IV-5 individuals are normal showing as wildtype while III-1, IV-1, IV-3, IV4 show heterozygous missense variant c.85652C $>$ G, p.(Pro28551Arg) in TTN gene.

(http://exac.broadinstitute.org/) database.

We found that proband III-1, IV-1, IV-3, IV-4 were heterozygous $\mathrm{m} / \mathrm{w}$ at this position while III-2, IV-2, IV-5 w/w were wild-type at this position. The mutation found results in a substitution of a p.(Pro28551 Arg) in the exon 326 of TTN gene in the affected father while other members IV-1, IV-3, IV-4 seem to be normal at this age as shown in figure 2. This mutation was further validated in 100 unrelated healthy persons, but no one has this sequence variation. The patient was treated with physiotherapy and genetic counseling was offered.

This study was approved by the Institutional Review Board (IRB) of King Abdullah International Medical Research Center (KAIMRC).

\section{Discussion}

In 2002, Hackman et al. reported the Finnish founder mutation (FINmaj: c.107780_107790delAAGTA ACATGGinsTGAAAGAAAAA, p.(Glu35927 Trp35930delinsValLysGluLys)), which is an $11 \mathrm{bp}$ insertion-deletion mutation exchanging 4 amino acids in the $363^{\text {rd }}$ and last exon of TTN (exon is called Mex6), which encodes the C-terminal immunoglobulin domain M10 of M-band in TTN (4). In addition to the FINmaj mutation, several other missense or truncating mutations have been associated with TMD in different European populations. These are located in the last 2 exons of TTN (Mex5 and Mex6). Previously, a heterozygous mutation in exon 292: c.68576C $>\mathrm{T}$ (p.Pro22859Leu) and a compound heterozygous mutation in exon 18: c.3100G $>$ A (p.Val1034Met); exon 240: c.49243G $>$ A (p.Ala16415Thr) was reported by Vasli et al. (5-8).

The same mutations that cause TMD, when present on both alleles, result in limb-girdle muscular dystrophy 2J (LGMD2J, MIM \#608807), in which most skeletal muscle groups are affected, leading to early disability. LGMD2J is a severe childhood-onset disease that causes proximal muscle weakness in the first or second decade and progresses to wheelchair confinement between 25-40 years of age. One patient homozygous for the truncating French TMD mutation c. $107890 \mathrm{C}>\mathrm{T}$ (p.Q35964*; NM_001267550.1) has also been reported with a proximal phenotype somewhat different from the homozygous Finnish LGMD2J patients (9). In the French patient, the disease onset was at 25 years, and the first muscle weakness occurred in the proximal upper limbs. Weakness and wasting progressed to all four limbs, and the patient lost ambulation at the age of 56 years. In addition, in consanguineous Chinese Han pedigree with autosomal recessive LGMD, a homozygous missense mutation TTN c. $107788 \mathrm{~T}>\mathrm{C}$, p. $($ Trp35930Arg) cosegregated with the disorder in the family and was absent from the ExAC control cohort (10).

TMD FINmaj mutation is predicted to cause cleavage of a larger part of TTN C-terminus, because immunofluorescence studies of homozygote LGMD2J muscles showed an absence of titin M8/M9 domain eoitopes (4). Also, Western blot analysis showed a severe reduction of the C-terminal TTN fragments in LGMD2J patient muscle (6). Loss of protein interactions of C-terminal TTN is thus likely consequence of the TMD/ LGMD2J mutations. Using yeast 2-hybrid analysis, Sarparanta et al. found that TTN containing TTN FINmaj mutation failed to interact with myospryn (11).

Ceyhan-Birsoy et al. (12) identified five individuals with childhood-onset centronuclear myopathy (CNM) who had compound heterozygous mutations in TTN. The patients presented with diffuse weakness, respiratory problems, occasional feeding difficulties and most had scoliosis and hypotonia. In one patient, both the maternally and paternally inherited mutation was a truncating change. Another patient was a compound heterozygote for a nonsense variant c.77989C $>\mathrm{T}$ (ex326) and a frameshift c.108114delA (ex326). Another patient, a 5-year-old boy, (979-1) had also two radical mutations inherited one from each parent, splice site mutation c. $15721+1 \mathrm{G}>\mathrm{A}$ and two frameshifts c.44998_45001del (ex358). None of the five patients had a noteworthy cardiac phenotype, although the ages at last examination only ranged from 5 to 19 years.

Muscle MRI is a very useful tool in patients 
with TMD and usually shows typical disease pattern characterized by dystrophic changes in the muscles of the anterior compartments of the lower legs starting in the anterior tibial muscle. Serum CK values are normal-mildly elevated, and there is no associated cardiomyopathy. The final diagnosis is confirmed by a genetic analysis of TTN gene (3). Next generation sequencing methods are extremely useful in the detection of genetic mutations in TMD, which are usually novel mutations and challenging to interpret. In silico prediction tools require a careful way of interpretation including functional studies, which are unfortunately not feasible with TTN due to its huge size that prevents the cloning and expression of the fulllength protein in in vitro systems (5).

In this study, we identified a heterozygous missense mutation in TTN gene c.85652C $>\mathrm{G}$, p.(Pro28551Arg) in a Saudi patient with bilateral facial weakness and scapular winging. This novel mutation in the TTN gene was suggested to be the genetic cause of the disease and further expanded the genetic spectrum of TTNassociated diseases in this family. This report adds to the literature a novel variant in TTN gene, which may be related to genes that cause the disease, but more case validation is needed. The novel mutation described in the present study widened the genetic spectrum of $T T N$-associated diseases, which may benefit studies addressing this disease in the future.

\section{References}

1. Krüger M, Kötter S. Titin, a central mediator for hypertrophic signaling, exercise-induced mechanosignaling and skeletal muscle remodeling. Front Physiol. 2016; 7:76.

2. Udd B, Partanen J, Halonen P, Falck B, Hakamies L, Heikkilä H, Ingo S, Kalimo H, Kääriäinen H, Laulumaa V, Tibial muscular dystrophy. Late adult-onset distal myopathy in 66 Finnish patients. Arch Neurol. 1993; 50:604-608.

3. Savarese M, Sarparanta J, Vihola A, Udd B, Hackman P. Increasing role of titin mutations in neuromuscular disorders. J Neuromuscul Dis. 2016; 3:293-308.
4. Hackman P, Vihola A, Haravuori H, Marchand S, Sarparanta J, De Seze J, Labeit S, Witt C, Peltonen L, Richard I, Udd B. Tibial muscular dystrophy is a titinopathy caused by mutations in TTN, the gene encoding the giant skeletal-muscle protein titin. Am J Hum Genet. 2002; 71:492-500.

5. Vasli N, Böhm J, Le Gras S, et al. Next generation sequencing for molecular diagnosis of neuromuscular diseases. Acta Neuropathol. 2012; 124:273-283.

6. Hackman P, Marchand S, Sarparanta J, Vihola A, Pénisson-Besnier I, Eymard B, Pardal-Fernández JM, Hammouda el-H, Richard I, Illa I, Udd B. Truncating mutations in $\mathrm{C}$-terminal titin may cause more severe tibial muscular dystrophy (TMD). Neuromuscul Disord. 2008; 18:922-928.

7. Van den Bergh PY, Bouquiaux O, Verellen C, Marchand S, Richard I, Hackman P, Udd B. Tibial muscular dystrophy in a Belgian family. Ann Neurol. 2003; 54:248-251.

8. Pollazzon M, Suominen T, Penttilä S, Malandrini A, Carluccio MA, Mondelli M, Marozza A, Federico A, Renieri A, Hackman P, Dotti MT, Udd B. The first Italian family with tibial muscular dystrophy caused by a novel titin mutation. J Neurol. 2010; 257:575-579.

9. Pénisson-Besnier I, Hackman P, Suominen T, Sarparanta J, Huovinen S, Richard-Crémieux I, Udd B. Myopathies caused by homozygous titin mutations: Limb-girdle muscular dystrophy $2 \mathrm{~J}$ and variations of phenotype. J Neurol Neurosurg Psychiatr. 2010; 81:1200-1202.

10. Zheng W, Chen H, Deng X, Yuan L, Yang Y, Song Z, Yang Z, Wu Y, Deng H. Identification of a novel mutation in the titin gene in a Chinese family with limbgirdle muscular dystrophy 2J. Mol Neurobiol. 2016; 53:5097-5102.

11. Sarparanta J, Blandin G, Charton K, Vihola A, Marchand S, Milic A, Hackman P, Ehler E, Richard I, Udd B. Interactions with M-band titin and calpain 3 link myospryn (CMYA5) to tibial and limb-girdle muscular dystrophies. J Biol Chem. 2010; 285:30304-30315.

12. Ceyhan-Birsoy O, Agrawal PB, Hidalgo C, et al. Recessive truncating titin gene, TTN, mutations presenting as centronuclear myopathy. Neurology. 2013; 81:1205-1214.

(Received April 26, 2019; Revised May 20, 2019; Accepted May 23, 2019) 